\title{
Phenotypic composition of salivary gland tumors: an application of principle component analysis to tissue microarray data
}

\author{
Hideto Iwafuchi ${ }^{1}$, Naoyoshi Mori², Takashi Takahashi ${ }^{3}$ and Yasushi Yatabe ${ }^{1}$ \\ ${ }^{1}$ Department of Pathology and Molecular Diagnostics, Aichi Cancer Center, Nagoya, Japan; ${ }^{2}$ First Department \\ of Pathology, Nagoya University School of Medicine, Nagoya, Japan and ${ }^{3}$ Division of Molecular Oncology, \\ Aichi Cancer Center Research Institute, Nagoya, Japan
}

\begin{abstract}
The tissue organization of the salivary gland is complex, and a large number of salivary gland tumor entities with a broad morphologic spectrum are listed, creating tumor classification schema for the salivary glands that are difficult to understand. In the present study, we attempted to examine how the anatomical components of the salivary gland are associated with morphological subtypes of tumors. We selected a panel of 12 molecules, which labeled one or some of the components, with all of the markers covering every component of the salivary glands. Using tissue microarray, expression profiles of these molecules were examined in four representative spots from each of $\mathbf{8 8}$ salivary gland tumors. The resulting large data matrix was analyzed using principle component analysis (PCA). We considered the first three eigenvectors to be significant; as the eigenvalues were more than 1.0 and the cumulative proportion achieved was $67 \%$. Comparison with expression patterns in normal tissue suggested that the three components represented myoepithelial differentiation, and luminal and basal cell phenotypes. Then, we compared the PCA results with individual morphologic subtypes. Individual subtypes were clustered among the three dimensions of the components. This implies that salivary gland tumors may be well characterized by using only three components.
\end{abstract}

Modern Pathology (2004) 17, 803-810, advance online publication, 16 April 2004; doi:10.1038/modpathol.3800122

Keywords: principle component analysis; tissue microarray; salivary gland tumor; immunohistochemical analysis

Salivary glands are anatomically composed of ducts and acini. The ducts are subdivided into intercalated, striated and interlobular ducts, in each of which basal cells at the outer side and luminal cells at the inner side are recognized. The acinar region consists of serous, mucinous and mixed glands, although the predominant gland type is, different among the three major salivary glands, that is, the parotid glands are rich in serous glands and the sublingual glands are rich in mucinous glands. The acini are lined by luminal cells, which are enclosed by myoepithelial cells. In addition to the complex tissue organization in the salivary glands, a large number of tumor entities have been listed, showing a broad range of morphologic and/or immunohistochemical features. One of the characteristics of salivary gland tumors is that although pleomorphic

Correspondence: Y Yatabe, MD, Department of Pathology and Molecular Diagnostics, Aichi Cancer Center, Kanokoden, Chikusa-ku, Nagoya 464-8681, Japan.

E-mail: yyatabe@aichi-cc.jp

Received 9 November 2003; revised 29 January 2004; accepted 2 February 2004; published online 16 April 2004 adenoma constitutes more than half of salivary gland tumors, the incidence of the other entities is evenly distributed. ${ }^{1}$ This is in contrast with breast cancers, which in the majority are ductal carcinomas, with mixed tumor, myoepithelioma and adenomyoepithelioma occurring rarely. Therefore, with salivary gland tumors, it is difficult to achieve a proper diagnosis occasionally, even using immunohistochemical analysis. In the present study, we attempted to examine how the anatomical components are associated with the morphological subtypes of salivary gland tumors.

For identification of each anatomical component, we used a panel of markers, each of which individually labeled one or some of the components, and together covered every component of the salivary glands. Although the expression patterns of CK7, CK8, CK14, CK19, $\alpha$-smooth muscle actin $(\alpha \mathrm{SMA})$, caldesmon and calponin have been reported already,,$^{2-7}$ there are only a few reports, or none, describing the expression of $\mathrm{p} 63,^{4,8}$ maspin, 14-3-3 $\sigma, \mathrm{CD}_{10}{ }^{9}$ and $\mathrm{CD} 44 \mathrm{v} 6^{10}$ in salivary gland tumors. Briefly, the putative functions of these molecules are summarized as follows. p63 is a 
homologue of p53 and has some relation to stem cell function. ${ }^{11-13}$ Maspin is a member of a serine proteinase inhibitor family (reviewed by Hendrix). ${ }^{14}$ Transfection of the maspin gene into a mammary carcinoma cell line promoted aggressiveness of the tumor cells through increased tumor growth, invasion and metastasis. ${ }^{15}$ Because maspin expression is observed in the myoepithelial cells of the breast, Sternlicht et $a l^{16}$ suggested that myoepithelial cells play a role as tumor suppressors. Hermeking et $a 1^{17}$ found that $14-3-3 \sigma$ is induced by $\gamma$-irradiation and by other DNA-damaging agents in a p53-dependent fashion, and that exogenous expression of 14-3-3 $\sigma$ causes G2 arrest in the colorectal cancer cell line, HCT116. Later studies revealed that 14-3-3 $\sigma$ sequesters the Cdc2/cyclin B1 complex in the cytoplasm, which prevents entry into the mitotic phase, thus causing mitotic catastrophe. ${ }^{18,19} \mathrm{CD} 10$ is an antigen well known as common acute lymphocytic leukemia antigen (CALLA), and recently, expression of this molecule has been reported in hepatocellular carcinoma, ${ }^{20}$ mesonephric tumors, ${ }^{20}$ trophoblastic tumors, ${ }^{20}$ breast cancers ${ }^{21}$ and endometrial stromal tumors. ${ }^{22}$ CD44 is a cellular adhesion molecule and a complex pattern of CD44 splice variants are expressed in various cancers (recently reviewed by Ponta et al). ${ }^{23}$ CD44v6 is one such variant, and its expression has been reported to be associated with more malignant behavior. ${ }^{24-26}$

Using these molecules, we describe here a novel approach in order to achieve the aim of this study. Principle component analysis (PCA) was applied to a large data matrix of expression profiles in 88 cases of salivary gland tumors, obtained by tissue microarray. Through shedding light on characteristics of expression profiles in individual subtypes, the results should give an overview of the salivary gland tumors.

\section{Materials and methods}

\section{Patients}

We studied a series of 88 patients with salivary gland tumors, selected from a file of the Department of Pathology and Molecular Diagnostics, Aichi Cancer Center Hospital. All the specimens were obtained by surgical resection, fixed with formalin and embedded in paraffin. The series included 21 cases of pleomorphic adenoma, 12 adenoid cystic carcinomas, 12 adenocarcinomas not otherwise specified (NOS), 11 Warthin's tumors, eight carcinomas ex pleomorphic adenomas (five duct carcinomas ex pleomorphic adenomas, three adenocarcinoma NOS ex pleomorphic adenomas), four salivary duct carcinomas, three mucoepidermoid carcinomas, two acinic cell carcinomas, and one each of basal cell adenoma, malignant myoepithelioma, oncocytic carcinoma and epithelialmyoepithelial carcinoma, in addition to 11 squamous cell carcinomas for comparison. The incidence of the subtypes was almost identical to that of the cohort observed during the past 3 years in our department, except that the number of pleomorphic adenomas examined in our series was reduced.

\section{Tissue Microarray}

In order to represent a whole feature, four spots were selected per tumor, and tissue microarrays were constructed with an MTA-1 manual tissue arrayer (Beecher Instruments, Inc., Silver Spring, MD, USA). Briefly, selected spots of the donor paraffin block were punched with a $0.6-\mathrm{mm}$ core needle, and transferred and arrayed in the recipient block using the arrayer. Then, serial $4-\mu \mathrm{m}$-thick sections on coated slide glasses were prepared for immunohistochemical analysis, similar to the procedure of a regular paraffin block. Verification of the tissue microarray has been described previously. ${ }^{27}$

\section{Immunohistochemistry}

Immunohistochemical examination proceeded according to the standard avidin-biotin-peroxidase complex method using the monoclonal antibodies listed in Table 1. Optimized dilution of primary antibodies and antigens retrieval method were noted in Table 1. In each batch of experiments, a slide microarrayed various tissues were stained as a control. Each section stained was evaluated semiquantitatively with the following criteria. A more than moderate intensity of signal was considered as positive and the proportion was scored as $0=$ negative; $1=$ less than $25 \%$ positive tumor cells; $2=26-50 \%$ positive tumor cells; $3=51-75 \%$ positive tumor cells; $4=$ more than $76 \%$ positive tumor cells. During the procedure, some of the tissue dots were noted to be missing and therefore 39 dots were excluded from the analysis.

\section{PCA}

In order to interpret the large data matrix of the expression profile with the molecules examined, we applied PCA to the matrix of about 4000 data points (88 cases $\times 4$ dots $\times 12$ molecules), using SYSTAT Software (SYSTAT Software Inc., Richmond, CA, USA) and Microsoft Excel (Microsoft, Bellevue, WA, USA). Briefly, based on original values, individual data was set among $n$-dimension space. The first component was given to maximize the variance of the data, and the second component was given to obtain the second largest variance of the data and makes a right angle with the first component. Similarly, this was followed by the third component. We considered that a component with an eigenvalue of more than 1.0 was a significant vector. 


\section{Results}

\section{PCA}

Using PCA, we analyzed the data matrix, which was obtained by four dots from each of the 88 salivary gland tumors, multiplexed by the 12 molecules examined. The results indicated that eigenvalues of the first three components were more than 1.0, and the cumulative proportion reached $67.1 \%$, indicating that the three components were able to represent most of the expression pattern of the individual dots (Table 2). Therefore, the three components were used for further analysis.

We attempted to gain an insight into the biological significance of each component. We focused on the molecules with absolute values of more than 0.5 in each component score (underlined, Table 2). The selected molecules shared an average of $70 \%$ of the cumulative component score. This implied that the molecules were representative of the components. With the criteria, all of the molecules examined could be divided into one of the components.
Caldesmon, $\alpha$ SMA, CD10, calponin and CD44v6 were selected as representative molecules for the first component, CK7, CK19 and CK8 for the second component, and p63, CK14, 14-3-3 $\sigma$ and maspin for the third component.

\section{First component}

In comparison with the staining pattern in the normal salivary glands, the molecules selected for the first component represented the feature of myoepithelial differentiation (Table 3). $\alpha$ SMA, caldesmon and calponin were positive in the myoepithelium from acini to intercalated ducts. Predominant expression of CD44v6 was seen in acinic cells, whereas basal-sided cells of acini and interlobular ducts were weakly positive. A weak CD10 signal was seen in the myoepithelium, whereas stromal fibroblasts were also positive in part.

\section{Second component}

The eigenvector of the second component was directed toward the phenotype of luminal cells.

Table 1 Antibodies used in the present study

\begin{tabular}{|c|c|c|c|c|}
\hline Antibody & Clone & Supplier & Dilution & Antigen retrieval \\
\hline$\alpha \mathrm{SMA}$ & $1 \mathrm{~A} 4$ & DAKO & $1: 50$ & Microwave \\
\hline p63 & $4 \mathrm{~A} 4$ & Pharmingen & $1: 200$ & Microwave \\
\hline Maspin & G167-70 & Pharmingen & $1: 150$ & Microwave \\
\hline CK8 & TS1 & Novocastra & $1: 100$ & Microwave \\
\hline CK7 & OV-TL20/30 & DAKO & $1: 25$ & Trypsin \\
\hline CK19 & b170 & Novocastra & $1: 100$ & Trypsin \\
\hline CK14 & LL002 & Novocastra & $1: 20$ & Microwave \\
\hline CD44v6 & F10 44-2 & Novocastra & $1: 100$ & Microwave \\
\hline CD10 & NCL-CD10 & Novocastra & $1: 40$ & Microwave in EDTA buffer \\
\hline Calponin & CALP & Novocastra & $1: 50$ & Trypsin \\
\hline Caldesmon & TD107 & Novocastra & $1: 50$ & Microwave \\
\hline $14-3-3 \sigma$ & Polyclonal (N-14) & Santa Cruz & $1: 150$ & Microwave \\
\hline
\end{tabular}

$\alpha \mathrm{SMA}, \alpha$-smooth muscle actin; CK, cytokeratin.

Table 2 Component scores for the molecules examined

\begin{tabular}{|c|c|c|c|c|c|}
\hline \multicolumn{2}{|c|}{ First component } & \multicolumn{2}{|c|}{ Second component } & \multicolumn{2}{|c|}{ Third component } \\
\hline Caldesmon & 0.829 & CK7 & 0.907 & $14-3-3 \sigma$ & 0.803 \\
\hline$\alpha \mathrm{SMA}$ & $\overline{0.815}$ & $\overline{\mathrm{CK} 19}$ & 0.869 & p63 & 0.758 \\
\hline$\overline{\mathrm{CD} 10}$ & 0.629 & $\overline{\mathrm{CK} 8}$ & 0.833 & CK14 & 0.709 \\
\hline$\overline{\text { Calponin }}$ & 0.577 & $\overline{\mathrm{CK} 14}$ & $-\overline{0.308}$ & Maspin & 0.682 \\
\hline CD44v6 & 0.537 & Maspin & -0.252 & $\overline{\mathrm{CD} 44 \mathrm{v} 6}$ & 0.407 \\
\hline$\overline{14-3-3 \sigma}$ & $-\overline{0.471}$ & p63 & -0.217 & CK8 & -0.265 \\
\hline Maspin & 0.406 & Caldesmon & -0.216 & CK7 & 0.228 \\
\hline p63 & 0.221 & $\alpha \mathrm{SMA}$ & 0.21 & Caldesmon & -0.191 \\
\hline CK19 & -0.218 & CD10 & -0.184 & CK19 & -0.095 \\
\hline CK14 & 0.176 & CD44v6 & -0.148 & Calponin & 0.078 \\
\hline CK8 & 0.075 & $14-3-3 \sigma$ & 0.057 & $\alpha \mathrm{SMA}$ & 0.065 \\
\hline CK7 & -0.017 & Calponin & 0.007 & CD10 & -0.01 \\
\hline Proportion & 24.07 & & 21.892 & & 21.094 \\
\hline Cumulative proportion & 24.07 & & 45.962 & & 67.056 \\
\hline
\end{tabular}

Underlines indicate the molecules which shared $70 \%$ or more of the cumulative component score in each component. 
Table 3 Expression patterns in the normal salivary gland

\begin{tabular}{|c|c|c|c|c|c|c|c|c|}
\hline & \multicolumn{2}{|c|}{ Acinar } & \multicolumn{2}{|c|}{ Intercalated } & \multicolumn{2}{|c|}{ Striated } & \multicolumn{2}{|c|}{ Interlobular } \\
\hline & Basal & Luminal & Basal & Luminal & Basal & Luminal & Basal & Luminal \\
\hline Caldesmon & ++++ & - & ++++ & - & + & - & - & - \\
\hline$\alpha \mathrm{SMA}$ & ++++ & - & ++++ & - & + & - & - & - \\
\hline CD10 & + & - & - & - & - & - & - & - \\
\hline Calponin & +++ & - & +++ & - & + & - & - & - \\
\hline CD44v6 & ++ & +++ & - & - & - & - & +++ & - \\
\hline CK7 & - & - & - & ++++ & - & ++++ & - & ++++ \\
\hline CK19 & - & - & - & ++ & - & ++++ & ++ & ++++ \\
\hline CK8 & - & ++++ & - & ++++ & - & ++++ & ++ & ++++ \\
\hline $14-3-3 \sigma$ & + & - & + & - & +++ & - & ++++ & - \\
\hline p63 & ++++ & - & ++++ & - & ++++ & - & ++++ & - \\
\hline CK14 & ++++ & - & ++++ & - & ++++ & - & ++++ & - \\
\hline Maspin & ++++ & - & ++++ & - & ++++ & - & ++++ & ++ \\
\hline
\end{tabular}

,$++++>75 \%$ of positive cells; +++, 50-75\%; ++, 25-50\%; +, <25\%; -, negative.

Luminal cells from intercalated ducts and acini were strongly positive for CK19, CK7 and CK8, while CK8 and CK19 were also positive in basal cells in the interlobular ducts.

\section{Third component}

For representative molecules of the third component, p63, CK14 and 14-3-3 $\sigma$ and maspin were selected. These molecules were expressed in basalsided cells throughout the salivary gland epithelium. All of these molecules were positive in the myoepithelium of the acinic region and the basal cells in the duct regions. Cells positive for 14-3-3 $\sigma$ were less frequent in the acini than in the duct regions.

Taken together, PCA categorized the expression profiles of the salivary gland tumors in the current series based on the presence or absence of myoepithelial differentiation, luminal cell phenotype and the features in the basal-sided cell (Figure 1). In other words, the expression profiles of the salivary glands tumors were characterized by these three features.

We then compared the results by PCA with individual morphological subtypes. As shown in Figure 1, each morphological subtype made clear clusters among the three dimensions, suggesting that the PCA results and morphological subtypes were well correlated. For example, the distribution of adenoid cystic carcinoma was restricted to the positive area of the first component and the negative area of the second components, while the values of the third component varied greatly. This implied that the carcinoma showed myoepithelial differentiation, but lacked the luminal phenotype, with varied expression of basal cell markers. In another example, salivary duct carcinoma demonstrated a lack of myoepithelial differentiation, a positive luminal cell phenotype, and negative expression of basal cell markers. This finding is compatible with the widely accepted concept on the origin or differentiation features of salivary duct carcinomas. The characteristics of individual morphologic subtypes are summarized in Table 4.

In terms of individual tumors, averaged standard deviations of the four dots from individual tumors were 0.51 for the first component, 0.92 for the second component and 0.61 for the third component, in contrast to the overall standard deviations of $1.75,1.68$ and 1.49 , respectively, indicating clustered distribution of the dots in the individual tumors. This implied that the expression profiles of individual cases were relatively uniform. However, there were some outliers. All four dots of a case of adenoid cystic carcinoma (ACC6) were distributed outside the cluster of the other adenoid cystic carcinomas (Figure 2). Histologically, this case was not a typical adenoid cystic carcinoma, but a poorly differentiated, solid variant. The other outlier was a case of adenocarcinoma NOS. This tumor phenotypically resembled a pleomorphic adenoma, but showed prominent nuclear atypia and infiltration of the surrounding fat tissue without capsule. The tumor could not be categorized to pleomorphic adenoma, and the morphology was very different from the other adenocarcinoma NOS, examined in this study. The diagnosis of low-grade adenocarcinoma NOS was finally made, but the feature of this tumor was quite unusual.

\section{Discussion}

In the current study, recently developed tissue microarray was used for the analysis. Tissue microarray has the advantage of simultaneous comparison of the expression patterns of many molecules in the same regions of tumors on the same slide. ${ }^{28}$ Simultaneous staining on the same slide reduces variation due to technical manipulations, and simultaneous evaluation facilitates the application of identical criteria throughout the subjects to be evaluated. Furthermore, the tissue array is beneficial 


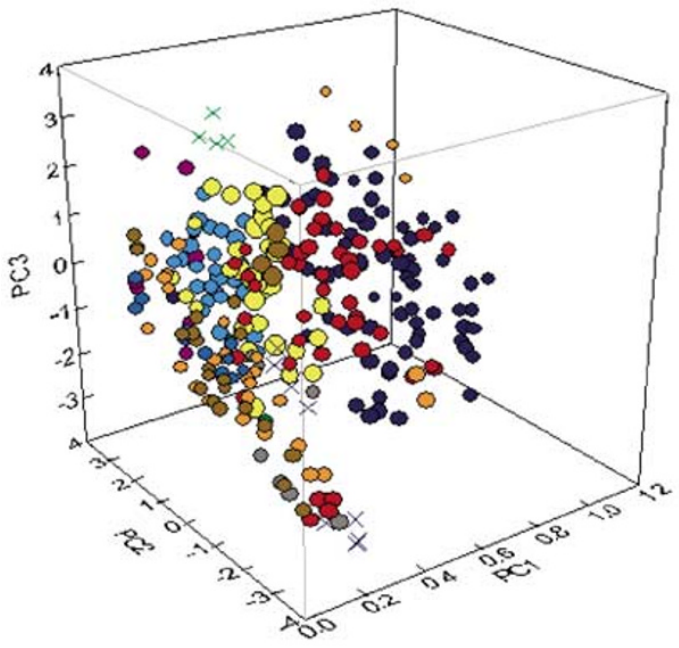

- Pleomorphic adenoma

Adenoid cystic carcinoma

- Adenocarcinoma, NOS

- Warthin's tumor

Squamous cell carcinoma

- Carcinoma ex pleomorphic adenoma

- Salivary duct carcinoma

- Mucoepidermoid carcinoma

- Acinic cell carcinoma

$\times$ Basal cell adenoma

$\times$ Malignant myoepithelioma

- Oncocytic carcinoma

$\times$ Epithelial-myoepithelial carcinoma
Adenoid cystic carcinoma

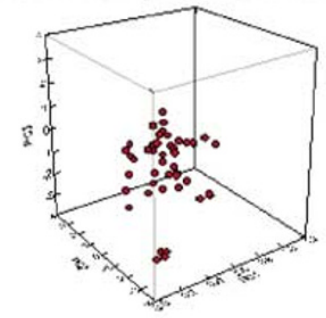

Salivary duct carcinoma
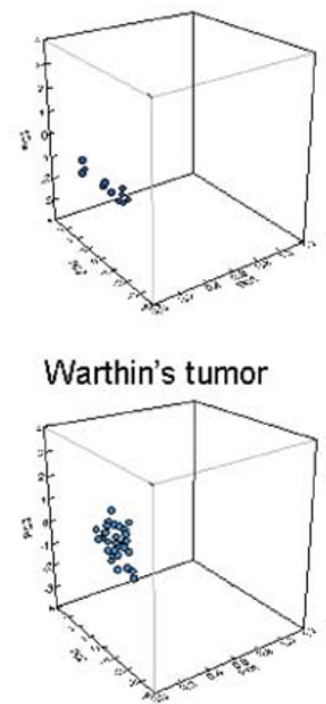

Pleomorphic adenoma

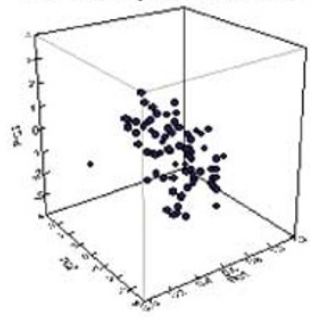

Carcinoma ex pleomorphic adenoma

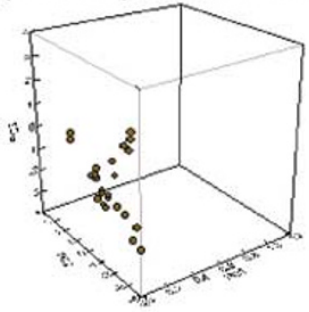

Mucoepidermoid carcinoma

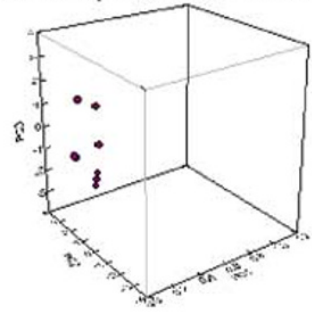

Figure 1 Scatter plots of the principle component scores of individual dots (left). Major six subtypes are displayed separately (right).

Table 4 Components and morphological subtypes

\begin{tabular}{lcccc}
\hline & $\mathrm{N}$ & $\begin{array}{c}\text { First component } \\
\text { Myoepithelial diff. }\end{array}$ & $\begin{array}{c}\text { Second component } \\
\text { Luminal phenotype }\end{array}$ & $\begin{array}{c}\text { Third component } \\
\text { Basal cellphenotype }\end{array}$ \\
\hline Pleomorphic adenoma & 21 & Yes & Variable & Variable \\
Adenoid cystic carcinoma & 12 & Yes & Yes & Variable \\
Adenocarcinoma, NOS & 12 & Variable & Yes & Yes \\
Warthin's tumor & 11 & No & No & Yes \\
Squamous cell carcinoma & 11 & No & Yes & Yo \\
Carcinoma ex pleomorphic adenoma & 8 & No & Yes & Vo \\
Salivary duct carcinoma & 4 & No & Variable & Yes \\
Mucoepidermoid carcinoma & 3 & No & No & Yes \\
Acinic cell carcinoma & 2 & Variable & Yes & No \\
Basal cell adenoma & 1 & Yes & No & No \\
Malignant myoepithelioma & 1 & No & No & \\
Oncocytic carcinoma & 1 & No & & \\
Epithelial-myoepithelial carcinoma & 1 & & & \\
\hline
\end{tabular}

in the case of heterogeneous staining pattern of the tumors, which may cause inter- and intra- observer differences. The region of an arrayed dot is small enough to reveal a single pattern of the staining, and heterogeneity is given by differences among the dots of the individual tumors. These advantages were exemplified by Torhorst et al, ${ }^{29}$ who reported better correlation of p53 staining pattern with clinical outcome by means of tissue microarray, than conventional whole section analysis. One concern may be whether a $0.6 \mu \mathrm{m}$ core of tissue used for the microarray can represent the whole tissue section and some articles have already addressed this issue. $^{30-32}$ A recent study by Camp et $a l^{32}$ showed that a two-fold redundancy per case can lead to more than $95 \%$ concordance between tissue microarrays 

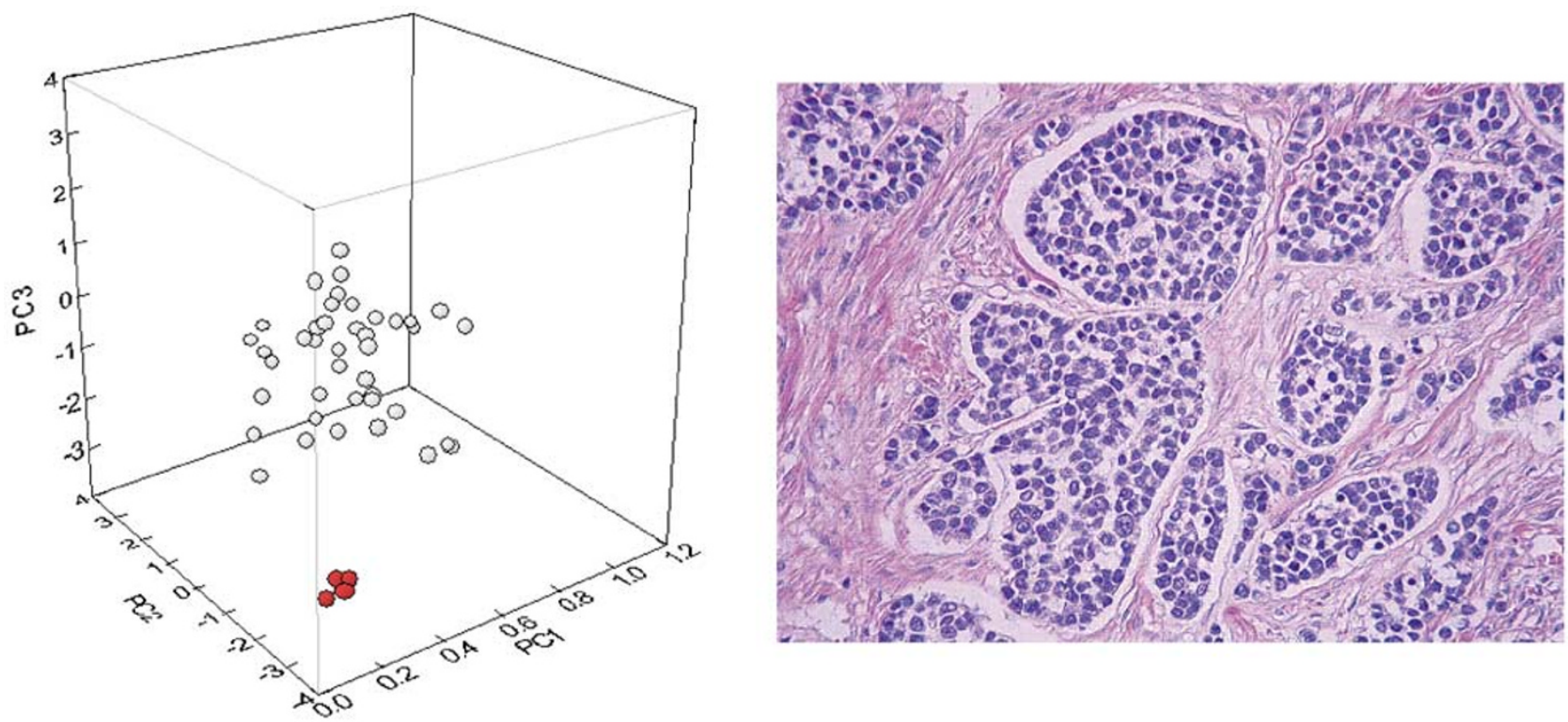

Figure 2 Example of an adenoid cystic carcinoma outlier. All of the four dots from ACC6 (in red) are apart from the others. The case shows the peculiar morphologic picture of a poorly differentiated, solid variant.

and whole tissue sections. ${ }^{32}$ Indeed, we recently found that maspin expression in non-small cell lung cancers was very heterogeneous, and tissue microarrays well represented this heterogeneity. ${ }^{33}$

Another benefit of using the tissue microarray is that it provides a large data matrix. Diagnosis of salivary gland tumors is very complex because the salivary gland is composed of various components and salivary gland tumors may be derived from a single component or a mixture of components. To understand this complexity, multivariate analysis is very helpful. The present study describes a novel approach, using one such multivariate analysis based on the large data matrix of expression profiles of salivary gland tumors.

PCA is a classical statistical method used to reduce the dimensions of variables. ${ }^{34}$ As it is difficult to compare the expression pattern of the 12 molecules simultaneously, PCA converts the reduced variables for ease of interpretation. Indeed, three principle components were extracted based on the characteristics of expression patterns using PCA. In comparison with the expression pattern of normal cells, the significance of the components could be accessed; myoepithelial differentiation as the first component, luminal cell phenotype as the second component, and a basal/stem cell phenotype as third component. According to the literature, the extraction was quite reasonable. All of the myoepithelium-associated markers examined make a single group of the first component. $\alpha \mathrm{SMA}$, calponin and caldesmon are well-known markers for normal and neoplastic myoepithelial cells, ${ }^{2,3,35}$ and CD44v6 expression of myoepithelium in the normal salivary glands has been reported. ${ }^{10}$

Current histological subclassification schemes for the salivary glands are largely based on putative normal counterparts of the tumors. ${ }^{36,37}$ For example, it is suspected that salivary duct carcinoma is derived from duct epithelium, while canalicular adenoma shows differentiation toward duct luminal epithelium. In the present study, the three components of PCA were obtained only by the expression status of the 12 molecules, and thus, the results of the PCA should be independent of morphological subtypes. However, the morphological subtypes were well clustered among three dimensions of the PCA characteristics. As shown in Table 4, each subtype showed distinct patterns. In a recent review, Zarbo $^{38}$ noted that the morphologic spectrum of salivary gland tumors comprised three basic cellular differentiations, that is, luminal, basal and myoepithelial cells, which are important for the biological interpretation of the salivary gland tumors. Most of the tumors keep within the spectrum between pure ductal luminal differentiation (canalicular adenoma) and pure myoepithelial differentiation (myoepithelioma). Our results are consistent with the concept proposed by Zarbo, and the current morphological subtypes are roughly characterized by the three components.

It is of note that markers of the third component label the basal-sided cells throughout the salivary glands. Histologically, the basal-side cells were divided into the myoepithelium and the duct basal cells, but the uniform expression pattern of multiple molecules appear to label a series of cells that share a certain function. p63, a homologue of p53, is one of the markers, and p63 is suspected to play a role in association with the stem cell function of the epithelium. ${ }^{13}$ In fact, p63-knockout mice display aphasia of the salivary glands. ${ }^{11,12}$ Regarding one of the other molecules, we have reported that $14-3-3 \sigma$ is upregulated during cell regeneration. ${ }^{27}$ Taken together, 
in addition to the sphincter function of acini, the myoepithelium may share a function with duct basal cells, which may be related to the stem cell function.

There appeared to be several findings in our study that were inconsistent with data in the literature. Although malignant myoepithelioma needs to have myoepithelial differentiation to establish the diagnosis, significant deviation of the first component, suggesting myoepithelial differentiation, was not revealed, as shown in Table 4. Similarly, epithelialmyoepithelial carcinoma showed little deviation of any of the components, whereas luminal and myoepithelial differentiation could be observed in the tumor. PCA results in Warthin's tumor also seem to disagree with the previous findings because of its deviation to basal phenotypes. This discordance may be explained by the trans-entity analysis in this study. In the raw data of myoepithelial carcinomas, actin and caldesmon were positive, but the extent was rather small in comparison with adenoid cystic carcinomas or some pleomorphic adenomas. Because PCA generates a functional equation with the staining score to maximize the variances of the dot-to-dot distances, it is possible that some principle components neglect small scores, and/or overestimate rare ones. The conversion leads to such artifacts, but the converted results may represent characteristics from a trans-entity view, that is, when a score is converted to a small value by the analysis, the conversion may suggest that the score is small enough to be negligible in the general schema of immunophenotypic profiles across salivary gland tumors.

\section{Acknowledgements}

We thank K Hayashi for excellent technical assistance, M Mabuchi and $\mathrm{H}$ Ishida for management of the paraffin blocks and slide preparation. This work is supported by a Grant-in-Aid for Encouragement of Young Scientists (B-15790195) from the Ministry of Education, Science, Sports and Culture, Japan.

\section{References}

1 Cheuk W, Chan JKC. Salivary gland tumors. In: Fletcher CDM (ed). Diagnostic Histopathology of Tumors. Churchill Livingstone: London, 2000, pp 231-311.

2 Prasad AR, Savera AT, Gown AM, et al. The myoepithelial immunophenotype in 135 benign and malignant salivary gland tumors other than pleomorphic adenoma. Arch Pathol Lab Med 1999;123:801-806.

3 Savera AT, Gown AM, Zarbo RJ. Immunolocalization of three novel smooth muscle-specific proteins in salivary gland pleomorphic adenoma: assessment of the morphogenetic role of myoepithelium. Mod Pathol 1997;10:1093-1100.

4 Weber A, Langhanki L, Schutz A, et al. Expression profiles of p53, p63, and p73 in benign salivary gland tumors. Virchows Arch 2002;441:428-436.

5 Ogawa Y, Toyosawa S, Ishida T, et al. Keratin 14 immunoreactive cells in pleomorphic adenomas and adenoid cystic carcinomas of salivary glands. Virchows Arch 2000;437:58-68.

6 de Araujo VC, de Sousa SO, Carvalho YR, et al. Application of immunohistochemistry to the diagnosis of salivary gland tumors. Appl Immunohistochem Mol Morphol 2000;8:195-202.

7 de Araujo VC, de Sousa SO. Expression of different keratins in salivary gland tumours. Eur J Cancer B Oral Oncol 1996;32B:14-18.

8 Bilal H, Handra-Luca A, Bertrand JC, et al. p63 is expressed in basal and myoepithelial cells of human normal and tumor salivary gland tissues. J Histochem Cytochem 2003;51:133-139.

9 Moritani S, Kushima R, Sugihara H, et al. Availability of CD10 immunohistochemistry as a marker of breast myoepithelial cells on paraffin sections. Mod Pathol 2002;15:397-405.

10 Fonseca I, Moura Nunes JF, Soares J. Expression of CD44 isoforms in normal salivary gland tissue: an immunohistochemical and ultrastructural study. Histochem Cell Biol 2000;114:483-488.

11 Yang A, Schweitzer R, Sun D, et al. p63 is essential for regenerative proliferation in limb, craniofacial and epithelial development. Nature 1999;398:714-718.

12 Mills AA, Zheng B, Wang XJ, et al. p63 is a p53 homologue required for limb and epidermal morphogenesis. Nature 1999;398:708-713.

13 Yang A, McKeon F. p63 and p73: p53 mimics, menaces and more. Nat Rev Mol Cell Biol 2000;1:199-207.

14 Hendrix MJ. De-mystifying the mechanism(s) of maspin. Nat Med 2000;6:374-376.

15 Zou Z, Anisowicz A, Hendrix MJ, et al. Maspin, a serpin with tumor-suppressing activity in human mammary epithelial cells. Science 1994;263: 526-529.

16 Sternlicht MD, Kedeshian P, Shao ZM, et al. The human myoepithelial cell is a natural tumor suppressor. Clin Cancer Res 1997;3:1949-1958.

17 Hermeking H, Lengauer C, Polyak K, et al. 14-3-3 sigma is a p53-regulated inhibitor of $\mathrm{G} 2 / \mathrm{M}$ progression. Mol Cell 1997;1:3-11.

18 Chan TA, Hermeking H, Lengauer C, et al. 14-3-3Sigma is required to prevent mitotic catastrophe after DNA damage. Nature 1999;401:616-620.

19 Lopez-Girona A, Furnari B, Mondesert O, et al. Nuclear localization of Cdc25 is regulated by DNA damage and a 14-3-3 protein. Nature 1999;397:172-175.

20 Ordi J, Romagosa C, Tavassoli FA, et al. CD10 expression in epithelial tissues and tumors of the gynecologic tract: a useful marker in the diagnosis of mesonephric, trophoblastic, and clear cell tumors. Am J Surg Pathol 2003;27:178-186.

21 Iwaya K, Ogawa H, Izumi M, et al. Stromal expression of CD10 in invasive breast carcinoma: a new predictor of clinical outcome. Virchows Arch 2002;440:589-593.

22 McCluggage WG, Sumathi VP, Maxwell P. CD10 is a sensitive and diagnostically useful immunohistochemical marker of normal endometrial stroma and of endometrial stromal neoplasms. Histopathology 2001;39:273-278.

23 Ponta H, Sherman L, Herrlich PA. CD44: from adhesion molecules to signalling regulators. Nat Rev Mol Cell Biol 2003;4:33-45.

24 Lipponen P, Aaltoma S, Kosma VM, et al. Expression of CD44 standard and variant-v6 proteins in transitional cell bladder tumours and their relation to prognosis during a long-term follow-up. J Pathol 1998;186:157-164. 
25 Soukka T, Salmi M, Joensuu H, et al. Regulation of CD44v6-containing isoforms during proliferation of normal and malignant epithelial cells. Cancer Res 1997;57:2281-2289.

26 Muller W, Schneiders A, Heider KH, et al. Expression and prognostic value of the CD44 splicing variants v5 and v6 in gastric cancer. J Pathol 1997;183:222-227.

27 Yatabe Y, Osada H, Tatematsu Y, et al. Decreased expression of 14-3-3sigma in neuroendocrine tumors is independent of origin and malignant potential. Oncogene 2002;21:8310-8319.

28 Kononen J, Bubendorf L, Kallioniemi A, et al. Tissue microarrays for high-throughput molecular profiling of tumor specimens. Nat Med 1998;4:844-847.

29 Torhorst J, Bucher C, Kononen J, et al. Tissue microarrays for rapid linking of molecular changes to clinical endpoints. Am J Pathol 2001;159:2249-2256.

30 Rubin MA, Dunn R, Strawderman M, et al. Tissue microarray sampling strategy for prostate cancer biomarker analysis. Am J Surg Pathol 2002;26:312-319.

31 Hoos A, Urist MJ, Stojadinovic A, et al. Validation of tissue microarrays for immunohistochemical profiling of cancer specimens using the example of human fibroblastic tumors. Am J Pathol 2001;158:1245-1251.
32 Camp RL, Charette LA, Rimm DL. Validation of tissue microarray technology in breast carcinoma. Lab Invest 2000;80:1943-1949.

33 Yatabe Y, Mitsudomi T, Takahashi T. Maspin expression in normal lung and non-small cell lung cancers: cellular property-associated expression under the control of promoter DNA methylation. Oncogene (in press).

34 Jolliffe IT. Principal Component Analysis, Springer Series in Statistics, 2nd edition. Springer: Berlin, 2002.

35 Grandi D, Campanini N, Becchi G, et al. On the myoepithelium of human salivary glands. An immunocytochemical study. Eur J Morphol 2000;38: 249-255.

36 Ellis GL, Auclair PL. Tumors of the salivary glands. Atlas of Tumor Pathology, 3rd edn. Armed Force Institute of Pathology: Washington DC, 1996.

37 Seifert G, Sobin LH. Histological typing of salivary gland tumours. World Health Organization International Histological Classification of Tumours, 2nd edn. Springer: Berlin, 1991.

38 Zarbo RJ. Salivary gland neoplasia: a review for the practicing pathologist. Mod Pathol 2002;15:298-323. 